\title{
ROSAT STUDIES OF EXTRAGALACTIC RADIO SOURCES
}

\author{
W. BRINKMANN, J. SIEBERT, W. YUAN \\ MPI für Extraterrestrische Physik \\ Postfach 1603 \\ D - 85740 Garching
}

\section{Introduction}

Complementary information over a large energy range appears to be the best way to study the class properties of Active Galactic Nuclei (AGN). While radio data provide superior spatial resolution required for the analysis of source structures and their morphology, optical data are a necessary ingredient for the classification of the objects and for the determination of their redshifts. Finally, X-ray (and more recently $\gamma$-ray) observations give vital information about the energetics of the central engines and on the physical conditions of the 'heart of the machine'.

With the recent advent of sensitive X-ray instruments like ROSAT and ASCA it became possible to study AGN in large numbers and at high redshifts. While ASCA has the best energy resolution achieved so far in the X-ray band, ROSAT provides the largest X-ray data base for the near future which consists of two parts:

- The ROSAT All-Sky Survey

- provides a nearly unbiased (apart from slightly varying exposure and variations in the Galactic absorption) coverage of the whole sky. The limiting sensitivity is a few $\times 10^{-13} \mathrm{erg} \mathrm{cm}^{-2} \mathrm{~s}^{-1}$ and the catalogue contains more than $\mathbf{6 0 . 0 0 0}$ sources.

- ROSAT Pointed Observations

- about 75.000 sources in $\sim 1.3$ sterad of the sky with very deep PSPC exposures $\left(\geq 2 \times 10^{-15} \mathrm{erg} \mathrm{cm}^{-2} \mathrm{~s}^{-1}\right)$. As serendipitous objects from pointed observations these sources have highly nonuniform exposures and selection biases. However, due to the generally long exposures more detailed spectral studies are possible. 


\section{The X-ray - radio data}

The sample of X-ray and radio - loud AGN was obtained by cross correlating large scale radio catalogs with the ROSAT source lists: in the northern sky the Greenbank $5 \mathrm{GHz}$ survey, in the southern sky the Molonglo Reference catalogue, and the $P M N$ radio survey. We thus obtain a list of $\approx 3700$ radio - loud X-ray sources of which nearly $2 / 3$ are optically unidentified. The results of broad band studies of the 'known' objects have been published elsewhere (Brinkmann et al. 1994, 1995).

In Fig. 1 we show the distribution of the sample in Galactic coordinates. The different types of sources are gray-coded with symbols proportional in size to their X-ray fluxes. Crosses represent the currently unidentified objects.

The more than 1400 unidentified sources on the northern sky have been re-observed with the VLA to obtain arcsec positions and core fluxes. With these positions we are able to find potential optical counterparts from digitized POSS plates and we can study the broad band properties of this population - however, without knowing the nature of the objects and their redshift (Brinkmann et al. 1996).

\section{3. 'Known' objects}

The number of 'known' radio - loud ROSAT detected AGN far exceeds all samples known previously. Therefore, statistically more reliable studies of different classes of objects like blazars, radio - loud quasars, radio galaxies and, amongst them, differentiations with respect to radio properties ( steep spectrum, flat spectrum) could be made. In Table 1 we give the currently known (approximate) numbers of objects for which $\mathrm{X}$-ray data are available.

TABLE 1. ROSAT detected radio sources

\begin{tabular}{lll}
\hline Type of Object & number & Remarks \\
\hline quasars & 654 & Survey: 360 \\
Radio galaxies & 245 & Survey only \\
blazars & 275 & HPQ: 63 \\
\hline
\end{tabular}

Indicated as well is the fraction of sources seen in the Survey only. Clearly, these numbers will increase in the future as more of the ROSAT sources will be identified optically.

With this large database one is able to study the X-ray as well as the broad band properties of various subgroups of objects. For example, assum- 


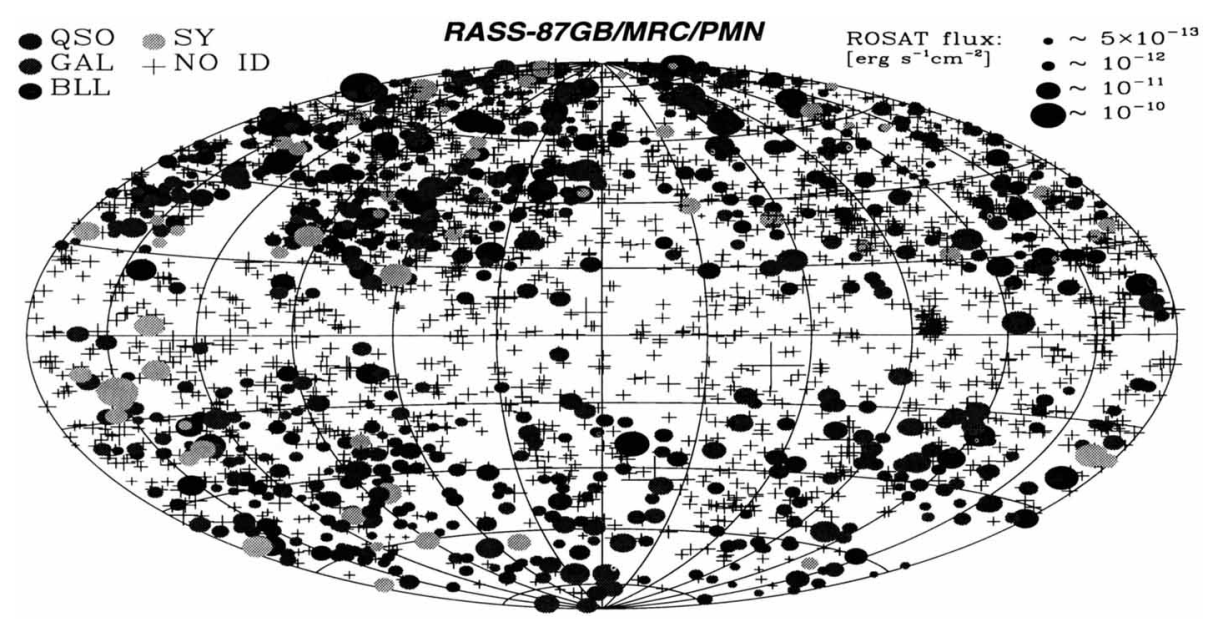

Figure 1. The ROSAT - radio sample of AGN plotted in Galactic coordinates. The symbol sizes are proportional to the X-ray flux of the objects. Crosses represent optically unidentified sources.

ing power law spectra either with fixed Galactic absorption or with free absorption the average spectral properties of the different object classes could be investigated using a maximum likelihood technique (Siebert 1996).

\begin{tabular}{r|rcc|rcr}
\hline \multirow{2}{*}{ AGN class } & \multicolumn{3}{|c|}{$\mathrm{N}_{H, \text { galactic }}$} & \multicolumn{4}{c}{$\mathrm{N}_{H, f r e e}$} \\
& $\mathrm{~N}$ & $\bar{\Gamma} \pm 1 \sigma$ & $\Sigma^{\text {intr }}$ & $\mathrm{N}$ & $\bar{\Gamma} \pm 1 \sigma$ & $\Sigma^{\text {intr }}$ \\
\hline Quasars & 197 & $2.14 \pm 0.06$ & $0.22 \pm 0.06$ & 165 & $2.20 \pm 0.21$ & $<0.34$ \\
Galaxies & 115 & $1.92 \pm 0.10$ & $0.47 \pm 0.08$ & 101 & $1.96 \pm 0.17$ & $<0.24$ \\
Seyferts & 41 & $2.02 \pm 0.14$ & $0.46 \pm 0.11$ & 37 & $2.04 \pm 0.24$ & $0.45 \pm 0.23$ \\
BL Lacs & 91 & $2.23 \pm 0.06$ & $0.25 \pm 0.06$ & 80 & $2.35 \pm 0.11$ & $<0.15$ \\
Unidentified & 938 & $2.23 \pm 0.04$ & $0.40 \pm 0.03$ & 815 & $2.34 \pm 0.11$ & $<0.17$ \\
\hline
\end{tabular}

Further, the ROSAT data base allows the investigation of well defined complete samples of objects. Even if a certain number of sources in a sample is not detected in X-rays the All - Sky Survey allows the determination of upper X-ray flux limits and the subsequent study of the sample using Survival Analysis techniques (p.e., the 2 Jy sample: Siebert et al. 1996). 


\section{4. 'Unknown' objects}

Optically not yet identified objects represent the largest group of sources in the sample. With accurate VLA positions, as noted above, we are able to find suitable optical counterparts from digitized POSS plates and, thus, optical fluxes.

In flux - flux diagrams, which are successfully used to classify objects and to attribute 'typical' flux values to these objects, the 'unknown' sources tend to fill in the gaps: these diagrams have intrinsic boundaries, not always obviously apparent, caused by the flux limits of the data sets used. As both, the radio as well as the ROSAT surveys reach much lower flux limits these boundaries are shifted or previously empty regions are now populated by ROSAT sources. This implies that some currently used criteria for source classifications might have to be revised: for example, the apparent gap between X-ray and radio selected BLLacs seems to be filled in by the 'unknown' objects. However, most of these sources need to be optically identified as BL Lacs.

On the other hand, unidentified sources placed in phase space volumes in these diagrams typical for certain classes of objects can be optically observed with high rate of success. For example, using the $\alpha_{r o}-\alpha_{o x}$ diagram for a pre - selection of BL Lac candidates the optical follow up observations reached success rates of more than about $30 \%$.

\section{Conclusions}

The cross-correlation of the ROSAT All-Sky Survey with large catalogs of radio sources yields a sample of in total more than 3700 radio-loud $X$-ray sources. This is the largest and least biased sample of radio-loud X-ray sources available. It allows the statistical analysis of $\mathrm{X}$-ray spectral properties and their dependence on redshift and radio properties for different AGN classes, the relation between soft $\mathrm{X}$-ray luminosity and the emission from other wavebands (radio, optical), the study of the X-ray luminosity function of radio-loud AGN and the connection between different classes of radio-loud AGN in terms of unification schemes. However, optical identifications for the majority of the objects are still needed to get a really unbiased (radio and X-ray) flux limited sample of AGN.

\section{References}

Brinkmann W., Siebert J., Boller Th., 1994, $A \& A, 281,355$

Brinkmann W., Siebert J., Reich W., et al, 1995, $A \& A S, 109,147$

Brinkmann W., Siebert J., Feigelson E., et al., 1996, $A \& A$ submitted

Siebert J., 1996, PhD thesis, Techn. University München

Siebert J., Brinkmann W., Morganti R., et al., 1996, MNRAS in press 